\title{
Evolution of the PVP-Pd surface interaction in nanoparticles through the case study of formic acid decomposition
}

\author{
Jaime García-Aguilar ${ }^{a}$, Miriam Navlani-García ${ }^{b}$, Ángel Berenguer-Murcia ${ }^{a}, K^{\prime}$ Kohsuke Mori ${ }^{b}$, \\ Yasutaka Kuwahara ${ }^{b}$,Hiromi Yamashita ${ }^{b,{ }^{*}}$, Diego Cazorla-Amorós ${ }^{a,{ }^{*}}$
}

${ }^{\text {a }}$ Materials Science Institute and Inorganic Chemistry Department, Alicante University, Ap. 99, E-03080 Alicante,
Spain.
b Division of Materials and Manufacturing Science, Graduate School of Engineering, Osaka University, 2-1 Yamada-
oka, Suita, Japan. Hydrogen production, palladium nanoparticles, formic acid decomposition, capping agent, PVP, surface interaction

\begin{abstract}
Palladium nanoparticles (Pd NPs) were synthesised by the reduction-by-solvent method using polyvinylpirrolidone (PVP) as capping agent. The non-static interaction between PVP and the metallic surface may change the properties of the NPs due to the different possible interactions, either through the $\mathrm{O}$ or $\mathrm{N}$ atoms of the PVP. In order to analyse these effects and their repercussion in their catalytic performance, Pd NPs with various PVP/Pd molar ratios $(1,10$ and 20) were prepared, deposited on silica and tested in the formic acid decomposition reaction. The catalytic tests were conducted using catalysts prepared by loading NPs with three different time lapses between their purification and their deposition on the silica support (1 day, 1 month, and 6 months). CO adsorption, FTIR spectroscopy, XPS and TEM characterisation were used to determine the accessibility of the Pd NPs surface sites, electronic state of Pd and the average NPs size, respectively. The $\mathrm{H}_{2}$ production from the formic acid decomposition reaction has a strong dependence with the Pd surface features, which in turn are related to the NPs aging time due to the progressive removal of the PVP.
\end{abstract}

\section{INTRODUCTION}

The use of capping agents, such as surfactants, organic ligands or dendrimers, is mandatory for the synthesis of stable colloidal metal nanoparticles (NPs), as they compensate the high surface energy of the NPs and protect them from aggregation. ${ }^{1,2}$ Nevertheless, it has also been reported that the capping agents used in colloidal synthesis are not merely stabilisers but also effective additives which modify the final NPs morphology features, not only in terms of NPs size and shape, ${ }^{3-6}$ but also of electronic properties. ${ }^{7-9}$ Thus, despite this necessity, the impact of these molecules once the metal NPs are synthesised is a

difficult issue, as it will affect the catalytic behaviour of metal-based catalytic systems by modifying either the NPs physicochemical properties or their accessibility by creating a physical barrier that hinders the access of reactants to the surface NPs catalytically active sites. ${ }^{10,11}$ In this respect, the accessibility to the NP surface is strongly dependent on the molecular structure of the capping agent, as it will determine its conformation once adsorbed on the metal surface. ${ }^{1,12,13}$

Due to its high protecting value, non-toxicity and solubility in many polar solvents, poly- $n$-vinylpyrrolidone (PVP) is one of the most common and widely studied capping agents in the synthesis of metal NPs. ${ }^{2,14-16}$ PVP has been proven to modify the NPs electronic properties through a charge transfer between the polymer functional groups and the metal surface. This was found for different metals NPs, such as $\mathrm{Pt},{ }^{7,17} \mathrm{Rh},{ }^{17} \mathrm{Au},{ }^{9} \mathrm{Ag}^{18,19}$ or Pd. ${ }^{8,20,21}$ Thus, due to the impact of the metal surface electronic modification in the catalytic performance of many PVP-capped NP-based systems, the interaction between chemisorbed PVP molecules and metal surfaces has been widely investigated but the nature of the interaction is still a focus of controversy. ${ }^{22}$ In this sense, some authors reported that PVP molecules anchor on the metal surface through its $\mathrm{O}$ atom, ${ }^{9,22}$ leading to the charge transfer from the metal surface to the polymer, while other authors claimed that the interaction is through the $\mathrm{N}$ atom, with the subsequent charge transfer from the PVP to the metal surface. ${ }^{1,21}$ Nevertheless, according to other studies found in the literature, the kind of interaction seems to depend on the metal NPs size. $^{21,23}$

Regarding the impact of this capping agent on the catalytic behaviour of the NPs, some authors reported the beneficial aspects of the PVP enhancing the catalytic proper- 
ties compared to pristine NPs due to the above mentioned electronic interactions..$^{9,11,20,24,25}$ However, studies found in the literature also addressed that the presence of PVP anchored to the NPs surface is responsible of the partial blocking of the surface Pd active sites. ${ }^{11,24,26}$ Both aspects are correlated due to their strong influence on the catalytic performance of the material but in most cases it is very difficult to differentiate their respective contribution. Along these lines, Zhao et al. ${ }^{24}$ and Tsunoyama et al. ${ }^{9}$ observed that when the NPs surface PVP coverage (term used by the authors to explain the coating degree of the $\mathrm{NP}$ by the capping agent) for their respective $\mathrm{Pd}$ and $\mathrm{Au}$ NPs, increases, the portion of accessible metal active sites decreases, while the specific catalytic activity of these highly protected sites is higher as compared to the less protected NPs.

The interactions between NPs and PVP molecules (electronic and anchoring/active sites blocking) cannot be considered as a static system even before or after purification. Some works reported the gradual and progressive dissolution of the capping agent, which initially was protecting the Pd NPs. ${ }^{27}$ During dissolution of the PVP the already mentioned changes in the NPs (electronic and anchoring/active sites blocking) can be modified therefore affecting their catalytic behaviour.

To evaluate this PVP-Pd interaction and study its evolution over time, a $\mathrm{Pd}$ surface-sensitive catalytic reaction can be used. ${ }^{28,29}$ For this purpose, together with the challenge of $\mathrm{H}_{2}$ production at ambient conditions by the catalytic decomposition of chemical compounds, formic acid (FA) decomposition in water solution has been selected as model reaction. Due to its safety properties, FA is one of the most promising chemical compounds to produce $\mathrm{H}_{2}$ near hydrogen consumption devices. Furthermore it is a nontoxic liquid at room temperature with a density of $\mathbf{1 . 2 2}$ $\mathrm{g} / \mathrm{cm}^{3}$, and can produce a gravimetric and volumetric $\mathrm{H}_{2}$ capacities of $4.4 \mathrm{wt} . \%$ and $5^{2} \mathrm{~g} / \mathrm{dm}^{3}$, respectively. ${ }^{30}$ Some noble metal-based catalysts (Ag, Pt and Pd) are the most selective towards $\mathrm{H}_{2}$ production instead of $\mathrm{CO}$ and water formation (the undesired possible reaction)..$^{3-33}$ Among them, Pd is one of the metals with the lowest activation energy and most promising TOF values. ${ }^{34,35}$ The incorporation of Pd NPs on different supports (carbon, zeolites, MOFs or silica) has also been studied in the literature with different results depending on the nature of the support and the active phases features. ${ }^{36-41}$

Herein the evolution of the PVP-Pd interaction over time as well as its repercussion on the final catalytic performance in the $\mathrm{H}_{2}$ production from the dehydrogenation of FA was assessed by synthesising a series of catalysts based on PVP-capped Pd NPs supported on a meso- and macroporous $\mathrm{SiO}_{2}$. To this end, NPs with different $\mathrm{PVP} / \mathrm{Pd}$ molar ratios and aging times were used. The relatively slow kinetic of this reaction and its strong dependence on the Pd surface features enables us to evaluate the pivotal role of the PVP-Pd interaction in the final catalytic performance.

\section{EXPERIMENTAL SECTION}

Synthesis of $\mathrm{SiO}_{2}$. The mesoporous silica support was prepared adapting a synthetic protocol previously described. ${ }^{42,43}$ For the synthesis, $0.400 \mathrm{~g}$ of macroporous porogen agent (Pluronic F127), $0.452 \mathrm{~g}$ of urea, and $5.052 \mathrm{~g}$ of acetic acid solution (o.o1 $\mathrm{M}$ ) were mixed under vigorous stirring for $80 \mathrm{~min}$, the final $\mathrm{pH}$ of the solution being around 4. Then the solution was cooled in an ice-water bath maintaining the stirring and the silica precursor (2.030 g TMOS) was added dropwise. This solution was kept under stirring for $40 \mathrm{~min}$ at $\mathrm{o}{ }^{\circ} \mathrm{C}$. The sol was introduced in a teflon-lined stainless steel autoclave and it was heated at $40{ }^{\circ} \mathrm{C}$ for $2 \mathrm{O} h$ to produce the aging of the sol (the $\mathrm{pH}$ after this step was around 4) followed by a hydrothermal treatment at $120{ }^{\circ} \mathrm{C}$ for $6 \mathrm{~h}$, to cause the urea decomposition (the final $\mathrm{pH}$ of the supernatant liquid was around $9-10)$. The final silica was then calcined at $550^{\circ} \mathrm{C}$ for $6 \mathrm{~h}\left(3^{\circ} \mathrm{C} / \mathrm{min}\right)$ to remove the surfactant.

Synthesis of Pd NPs. Colloidal Pd NPs were prepared by a reduction by solvent method reported by our research group. ${ }^{42,44-46}$ The $\mathrm{Pd}$ precursor was palladium acetate $\left(\mathrm{Pd}(\mathrm{OAc})_{2}, 98 \%\right.$, Sigma-Aldrich $)$ and the capping agent used for the synthesis was polyvinylpyrrolidone (PVP 30 $\mathrm{K}$, Sigma-Aldrich). The preparation was carried out in an inert atmosphere using a Schlenk system, according to the following procedure. For solution 1, the necessary amount of PVP (three PVP/Pd molar ratios were prepared, 1, 10 and 20) was added to $60 \mathrm{ml}$ of ethylene glycol in a three-neck round-bottom flask and the solution was stirred at $80{ }^{\circ} \mathrm{C}$ for $2 \mathrm{~h}$ using a magnetic stirrer. For solution 2, $0.112 \mathrm{~g}(0.5 \mathrm{mmol})$ of $\mathrm{Pd}(\mathrm{OAc})_{2}$ were dissolved in 12 $\mathrm{ml}$ of 1,4-dioxane by stirring for $2 \mathrm{~h}$ at room temperature using also a magnetic stirrer. The color of this solution was light orange.

After that, solution 1 was cooled down to o ${ }^{\circ} \mathrm{C}$ in an ice bath and $1 \mathrm{M}$ of $\mathrm{NaOH}$ solution was added under stirring in order to adjust the $\mathrm{pH}$ to 10 . Then, solution 2 was poured into solution 1 under vigorous stirring in order to ensure homogenization and the final mixture was heated up to $100{ }^{\circ} \mathrm{C}$. The solution changed its color from light orange to dark brown, which indicated that the metallic colloid had been formed. Then, the temperature was maintained for $2 \mathrm{~h}$, time after which the bath was removed and the colloidal suspension was cooled down to room temperature.

Once the colloids were prepared, the Pd NPs were purified and redispersed in methanol. The purification was carried out by pouring an aliquot of NPs colloid into a glass bottle, adding an excess of acetone and shaking the solution. Then, the supernatant organic phase was removed and the purified NPs were redispersed by stirring in a known amount of methanol, so that the final Pd concentration was perfectly known. Once the NPs are purified, they can be stored under ambient conditions for long periods of time while retaining their stability against sintering. In this study, the colloids were stored in a glass bottle for 1 and 6 month (denoted as "aging times" in this study), for their further impregnation onto $\mathrm{SiO}_{2}$ support. 
Support impregnation. The silica support was impregnated by following an impregnation in excess volume method with the colloidal methanol suspension of NPs for 2 days under magnetic agitation, with the adequate volume of metallic colloids so as to achieve a 1 wt. \% Pd loading. For each PVP/Pd molar ratio (1, 10 and 20), three samples were prepared by using different NPs aging times, fresh NPs, 1 month and 6 months of aging after the purification step, respectively, so as to have a total of 9 samples. After the impregnation step, the solvent was removed by heating at $65^{\circ} \mathrm{C}$ under stirring. The resulting catalysts were denoted as follow:

$$
\mathrm{Pd}_{\text {aging time }}^{\mathrm{PVP} / \mathrm{Pd}^{\text {ratio }}} / \mathrm{SiO}_{2} \text {. }
$$

A Pd catalyst on silica (1 wt. \%) was prepared as a reference sample by the standard impregnation method and using $\mathrm{Pd}(\mathrm{OAc})_{2}$ as precursor for comparison purposes.

Formic acid decomposition tests. The catalytic tests were conducted in a Pyrex reaction vessel $(30 \mathrm{ml})$ sealed with a rubber septum and under magnetic agitation. For the standard catalytic tests, $50 \mathrm{mg}$ of powder sample and $9.6 \mathrm{ml}$ of water were introduced in the vessel. In order to disperse the sample and obtain an inert atmosphere, the catalyst-water suspension was treated in an ultrasound bath for 15 min and subsequently bubbled with argon gas for 30 min. $0.39 \mathrm{ml}$ of FA were added (final FA concentration $1 \mathrm{M}$ in water) to the vessel and it was placed in an oil bath at $30{ }^{\circ} \mathrm{C}$ with magnetic stirring for $3 \mathrm{~h}$. The $\mathrm{H}_{2}$ output was monitored periodically by using a Shimadzu $\mathrm{GC}_{14} \mathrm{~B}$ equipped with $\mathrm{MS}_{5} \mathrm{~A}$ column. For this purpose, injections in the GC were made and the resulting peak areas were converted into $\mathrm{H}_{2}$ concentration by using a calibration with the corresponding standard gas. The selectivity of the reaction was evaluated by checking the $\mathrm{CO}$ generated at the end of the experiments. The TOF $\left(h^{-1}\right)$ was calculated using the following equation:

$$
\text { TOF }\left(h^{-1}\right)=\frac{\text { produced } \mathrm{H}_{2}(\mathrm{~mol})}{\text { Pd content }(\mathrm{mol}) \times \text { time }(\mathrm{h})}
$$

where the produced $\mathrm{H}_{2}(\mathrm{~mol})$ was measured at a reaction time of $15 \mathrm{~min}$ and the Pd content (in mol) is the total number of moles of Pd added to each reaction batch, taking into account the catalyst weight and the metal loading determined by ICP-OES.

Catalysts characterisation. The mesoporous silica support and the catalysts were characterised by $\mathrm{N}_{2}$ adsorption-desorption at $-196{ }^{\circ} \mathrm{C}$ and by $\mathrm{CO}_{2}$ adsorption at $\mathrm{o}^{\circ} \mathrm{C}$ (Quantachrome, Autosorb 6B) to analyse the porous texture of the materials. Apparent surface area values were calculated from $\mathrm{N}_{2}$ adsorption isotherms using the BET equation $\left(\mathrm{S}_{\mathrm{BET}}\right)$. Total micropore volume $\left(\mathrm{V}_{\mathrm{DR}}\left(\mathrm{N}_{2}\right)\right)$ and narrow micropore volume $\left(\mathrm{V}_{\mathrm{DR}}\left(\mathrm{CO}_{2}\right)\right)$ were calculated applying the Dubinin-Radushkevich (DR) equation to the $\mathrm{N}_{2}$ adsorption data at $-196{ }^{\circ} \mathrm{C}$ and the $\mathrm{CO}_{2}$ adsorption data at $\mathrm{o}{ }^{\circ} \mathrm{C}$, respectively.

The catalysts were analysed by Fourier Transform Infrared Spectroscopy (FTIR-670o, JASCO) in transmittance mode in vacuum conditions. All samples were diluted using the same catalyst: $\mathrm{KBr}$ weight ratio (1:10o). In order to obtain comparable results, a portion of the mixture (for each one of the samples analysed) was ground in an agate mortar to homogenise it and then, a cylindrical pellet was obtained in a manual mounting press for analysis. This technique is useful to analyse the typical IR-bands of the PVP and their evolution over time.

The Pd loading in the catalysts was determined by inductively coupled plasma-optical emission spectroscopy (ICPOES), in a Perkin-Elmer Optima 4300 system. The extraction of the metal was made by treating the samples with aqua regia at room temperature and adjusting the $\mathrm{Pd}$ concentration from o to $20 \mathrm{ppm}$. The samples were also characterised by Transmission Electron Microscopy (TEM) with a JEOL JEM-2010 microscope operating at 200 $\mathrm{kV}$ with a space resolution of $0.24 \mathrm{~nm}$. For the analysis, a small amount of sample was suspended in a few drops of hexane, and sonicated for few minutes. A drop of this suspension was then deposited onto a 300 mesh Lacey copper grid and left to dry at room temperature. TEM analyses allowed the determination of the average metal particle size by counting at least $100 \mathrm{NPs}{ }^{46}$

$\mathrm{CO}$ adsorption experiments for the as-prepared catalysts were performed in a BELCAT-M automatic device (BEL JAPAN, INC) which had an experimental error of $3 \%$. For each measurement, $30 \mathrm{mg}$ of catalyst were added in a quartz tube reactor. Different experiments were conducted to determine the $\mathrm{CO}$ adsorbed at $50{ }^{\circ} \mathrm{C}$ per mol of $\mathrm{Pd}$. As the Pd NPs are partially surrounded by PVP molecules, these experiments can provide an estimation of the $\mathrm{Pd}$ accessibility.

The electronic states of the elements in the samples were determined by X-ray Photoelectron Spectroscopy (XPS) using a K-Alpha of Thermo-Scientific spectrometer, equipped with an $\mathrm{Al}$ anode. Characterisation of the electronic states of each element was made from the relative area of the peaks resulting in the $3 \mathrm{~d}$ and is region of $\mathrm{Pd}$ and $\mathrm{N}$ spectrum, respectively.

\section{RESULTS AND DISCUSSION}

Formic acid decomposition tests. All the catalysts (with different PVP/Pd ratio and aging time in methanol solution) were tested in the FA decomposition reaction as described in the experimental section. It must be noted that no induction time (i.e. $\mathrm{H}_{2}$ was immediately produced from the beginning of the reaction) for any of the catalysts tested in this study under the experimental conditions used. In Figure 1 and Table 1 , the $\mathrm{H}_{2}$ production at 30 ${ }^{\circ} \mathrm{C}$ after $18 \mathrm{o} \mathrm{min}$ is presented. In order to evaluate the effect of the aging time, the loss of catalytic activity (as compared to the activity of the fresh samples) is also listed in Table 1 , together with the TOF values. It is worth mentioning that in all cases the selectivity towards the $\mathrm{H}_{2}$ generation was almost $100 \%$, as no $\mathrm{CO}$ was detected at the end of the catalytic tests. The control experiments conducted with the bare support did not generate any $\mathrm{H}_{2}$ gas. 


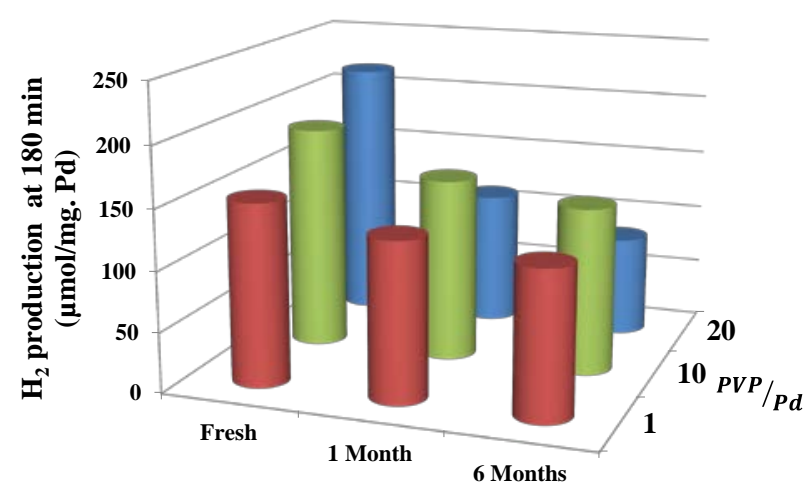

Figure 1. $\mathrm{H}_{2}$ production after $180 \mathrm{~min}$ of reaction at $30^{\circ} \mathrm{C}$ for all the studied $\mathrm{Pd} / \mathrm{SiO}_{2}$ catalysts normalised by the $\mathrm{Pd}$ weight.

Table 1. Catalytic results of FA decomposition at $30{ }^{\circ} \mathrm{C}$ after $180 \mathrm{~min}$ of reaction and the loss of catalytic activity respect to the counterpart fresh catalysts.

$\begin{array}{cccc}\text { Sample } & \mathrm{H}_{2} \text { production at } & \text { Loss of } & \text { TOF }\left(\mathrm{h}^{-}\right. \\ 180 \mathrm{~min}(\mu \mathrm{mol} / \mathrm{mg} & \begin{array}{c}\text { activity }(\%) \\ {[\mathrm{b}]}\end{array} & \left.{ }^{1}\right)^{[\mathrm{c}]} \\ & \mathrm{Pd})^{[\mathrm{a}]} & & \end{array}$

\begin{tabular}{|c|c|c|c|}
\hline $\mathrm{Pd}_{\mathrm{F}}^{1} / \mathrm{SiO}_{2}$ & 151.7 & - & 17.2 \\
\hline $\mathrm{Pd}_{1}^{1} / \mathrm{SiO}_{2}$ & 132.6 & 12.5 & 16.7 \\
\hline $\mathrm{Pd}_{6}^{1} / \mathrm{SiO}_{2}$ & 122.6 & 19.1 & $15 \cdot 7$ \\
\hline $\mathrm{Pd}_{\mathrm{F}}^{10} / \mathrm{SiO}_{2}$ & 186.5 & - & 18.4 \\
\hline $\mathrm{Pd}_{1}^{10} / \mathrm{SiO}_{2}$ & 152.4 & 18.3 & 17.4 \\
\hline $\mathrm{Pd}_{6}^{10} / \mathrm{SiO}_{2}$ & 139.1 & 25.4 & 16.9 \\
\hline $\mathrm{Pd}_{\mathrm{F}}^{20} / \mathrm{SiO}_{2}$ & 217.8 & - & 23.4 \\
\hline $\mathrm{Pd}_{1}^{20} / \mathrm{SiO}_{2}$ & 152.4 & 48.6 & 16.2 \\
\hline $\mathrm{Pd}_{6}^{20} / \mathrm{SiO}_{2}$ & 83.9 & 61.5 & 12.3 \\
\hline \multicolumn{4}{|c|}{$\begin{array}{l}\text { [a] Normalised } \mathrm{H}_{2} \text { production per } \mathrm{mg} \text { of } \mathrm{Pd} \text { calculated by } \\
\text { ICP-OES results (vide infra). }\end{array}$} \\
\hline \multicolumn{4}{|c|}{$\begin{array}{l}\text { [b] Catalytic activity decay (\%) with respect to the fresh } \\
\text { catalyst counterpart. }\end{array}$} \\
\hline
\end{tabular}

The data plotted in Figure 1 revealed that the catalytic activity towards $\mathrm{H}_{2}$ production showed a very important dependence on both $\mathrm{PVP} / \mathrm{Pd}$ ratio and aging time. As it can be seen, for the three $\mathrm{PVP} / \mathrm{Pd}$ ratios under investigation, the largest $\mathrm{H}_{2}$ production was achieved for the fresh samples and a progressive decay of the catalytic activity was appreciated with the aging time ( 1 and 6 months), being much more pronounced in the case of the catalysts prepared from the colloid with a PVP/M ratio of 20. As it can be seen in Table 1, samples prepared from aged col- loids with $\mathrm{PVP} / \mathrm{Pd}$ ratio of 20 , suffered a dramatic catalytic activity decay ( 48.6 and $61.5 \%$ on the basis on the fresh sample, for aging times of 1 and 6 months, respectively), while more gradual decays were detected for the other two PVP/Pd ratios investigated. Concerning the PVP content effect, it was observed that the $\mathrm{H}_{2}$ produced by the fresh samples was higher as the PVP used for the colloidal synthesis increases $(151.7,186.5$ and $217.8 \mu \mathrm{mol}$ of $\mathrm{H}_{2} / \mathrm{mg}$. Pd for the fresh samples with PVP/Pd ratios of 1 , 10 and 20, respectively). These catalytic activity trends correlated well with the initial TOF values (calculated on the basis of Pd loading) for the three sets of catalysts.

On the other hand, for the Pd impregnated sample only $20.4 \mu \mathrm{mol}$ of $\mathrm{H}_{2} / \mathrm{mg}$ Pd were obtained after $180 \mathrm{~min}$ of reaction. This $\mathrm{H}_{2}$ production is very low compared with the PVP capped Pd NPs, which evidenced the potential beneficial effects of the capping agent in catalyst synthesis, as it was previously reported elsewhere. ${ }^{9,24}$

The strong dependence of the catalytic performance on the PVP/Pd ratio and aging time might be related to the Pd NPs characteristics, in terms of catalytic sites accessibility, NPs size, or electronic features. Then, to elucidate the influence of the studied parameters in the catalytic behaviour towards the $\mathrm{H}_{2}$ production from the FA dehydrogenation, further characterisation results are included in the following section.

Characterisation of the catalysts. The textural analysis of the pure $\mathrm{SiO}_{2}$ used as support shows a combination of type I and type IV isotherms, typical of mesoporous materials with a hysteresis cycle and a certain degree of microporosity inherent to the silica. The $\mathrm{SiO}_{2}$ presented a specific surface area of $\sim 230 \mathrm{~m}^{2} / \mathrm{g}$ calculated by the BET method, with a pore size distribution ranging from 5 to 20 $\mathrm{nm}$ (with the maximum at around $16 \mathrm{~nm}$ ) calculated by the $\mathrm{BJH}$ model applied to the desorption branch of the isotherm. The mesoporous $\mathrm{SiO}_{2}$ has a very low specific micropore volume of $0.09 \mathrm{~cm}^{3} / \mathrm{g}$ in $\mathrm{N}_{2}$ adsorption according to the Dubinin-Radushkevich method. The $\mathrm{SiO}_{2}$ used as support of the catalysts also showed macroporosity produced during the hydrothermal treatment at $120{ }^{\circ} \mathrm{C}$. This macroporosity is due to the interparticle gaps between the $\mathrm{SiO}_{2} \mu$-spheres (between 4-6 $\mu$ m of diameter) formed by the spinodal decomposition during the hydrothermal step. ${ }^{47}$ No significant changes in the textural properties were observed after the impregnation of the $\mathrm{Pd}$ NPs in any catalyst.

In order to analyse the PVP presence in the Pd based catalysts, all the catalysts, the pure $\mathrm{SiO}_{2}$ and the raw PVP were submitted to FTIR analysis. The results are presented in the Figure 2. 

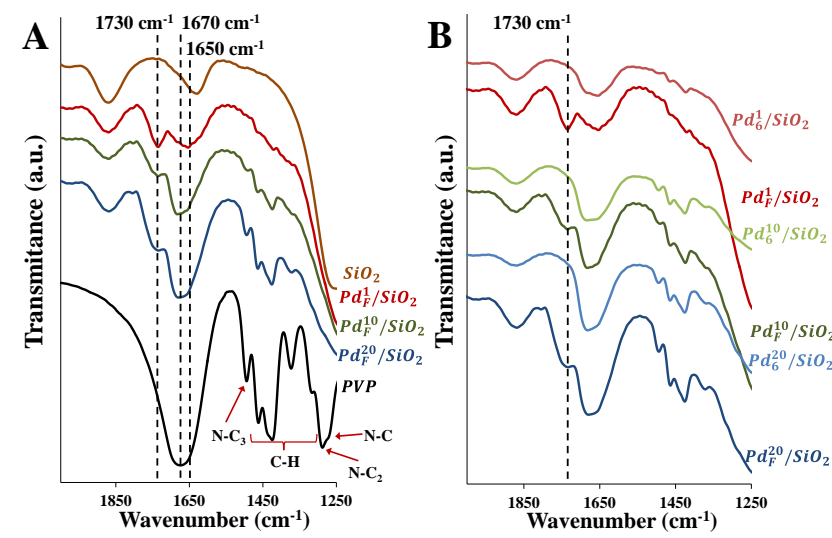

Figure 2. Transmitance FTIR spectra of the main catalysts prepared in this work. A) Comparison between the fresh catalysts, $\mathrm{SiO}_{2}$ support and raw PVP. B) FTIR spectra of the fresh samples and the 6 months aged counterparts.

In Figure 2A, the FTIR spectrum of the pure PVP is presented and its characteristic bands can be observed; around $1280 \mathrm{~cm}^{-1}$ the two stretching vibrations of the two $\mathrm{N}-\mathrm{C}$ bonds, between 1300 and $1480 \mathrm{~cm}^{-1}$ the typical $\mathrm{C}-\mathrm{H}$ vibrations of the PVP, close to $1500 \mathrm{~cm}^{-1}$ the $\mathrm{N}-\mathrm{C}$ stretching of the $\mathrm{N}-\mathrm{C}=\mathrm{O}$ group and finally the most intense band corresponding to the $\mathrm{C}=\mathrm{O}$ stretching at $1660-1675 \mathrm{~cm}^{-1}$. ${ }^{21}$ At the top of the plot, the FTIR spectrum of the $\mathrm{SiO}_{2}$ used as support of the Pd NPs is also shown. A very strong absorption of the pure $\mathrm{SiO}_{2}$ is observed at around 1000$1300 \mathrm{~cm}^{-1}$ due to its fundamental framework vibrational modes. Two more bands are observed in the FTIR spectrum located at 1630 and $1860 \mathrm{~cm}^{-1}$. The band located at $1630 \mathrm{~cm}^{-1}$ can be assigned to bending vibration of water molecules and the other band corresponds to bulk Si-O combination mode. ${ }^{48}$ For the fresh catalysts represented in Figure $2 \mathrm{~A}$, it is possible to observe the presence of the characteristic PVP signals but it is quite difficult to differentiate the bands under $1300 \mathrm{~cm}^{-1}$ due to the high intensity of the $\mathrm{SiO}_{2}$ bands. It is possible to see that the intensity of the PVP bands in the fresh catalysts is proportional to the amount PVP used in the synthesis of the NPs.

Some modifications in the FTIR spectra are observed in the fresh catalysts with respect to pure PVP. The presence of a new band located at around $1730 \mathrm{~cm}^{-1}$ in all the samples and a slight shift of the band of the carbonyl group $\left(1650 \mathrm{~cm}^{-1}\right)$ to lower wavenumber values can be observed which is clearly observed for the sample with the lowest amount of PVP $\left(\mathrm{Pd}_{\mathrm{F}}^{1} / \mathrm{SiO}_{2}\right)$. As it is explained in the introduction, the previously reported interaction between Pd and carbonyl group of the PVP molecules is based in a charge transfer from metal to the carbonyl group. This interaction decreases the carbonyl bond strength displacing the typical IR band to slightly lower wavenumber values. In this sense, different carbonyl species (interacting and non-interacting with the metal NPs surface) might be present in the catalysts. One possibility according to a previous study in similar materials ${ }^{27}$ is that the band at $1650 \mathrm{~cm}^{-1}$ (shoulder in the $\mathrm{Pd}_{F}^{20} / \mathrm{SiO}_{2}$ and
$\mathrm{Pd}_{F}^{10} / \mathrm{SiO}_{2}$ case but band in the $\left.\mathrm{Pd}_{F}^{1} / \mathrm{SiO}_{2}\right)$ could be ascribed to the $\mathrm{CO}$ stretching of the pyrrolidone rings interacting with the Pd on the NPs surface and the band of the non-interacting $\mathrm{CO}$ was located at $1670 \mathrm{~cm}^{-1}$ (as in the pure PVP). In our fresh catalysts, both bands are observed and when the amount of used PVP in the synthesis is reduced to 1 the band located at $1650 \mathrm{~cm}^{-1}$ (associated to the Pd species interaction with the carbonyl groups of the PVP) is clearly observed. The assignation of the band located at $1730 \mathrm{~cm}^{-1}$ is quite controversial; some studies ${ }^{21}$ have assigned this band to the $\mathrm{O}=\mathrm{C}-\mathrm{O}$ (ester groups) formed when the PVP ring is broken due to the bridged interaction with $\mathrm{Pd}$ particles with sizes over $10 \mathrm{~nm}$ or some ring opening due to the basic conditions used in the synthesis. From our earlier observations ${ }^{27}$, another possible assignment for the band at $\sim 1730 \mathrm{~cm}^{-1}$ might be the $\mathrm{C}=\mathrm{O}$ stretching of the pyrrolidone rings directly interacting with the metal surface.

On the other hand, as the aging time (time during which the colloid is suspended in methanol) increases some changes in the FTIR spectra are observed. For the sake of brevity, only the spectra of the fresh and 6 months aged samples are plotted in Figure $2 \mathrm{~B}$. In the region of $\mathrm{C}-\mathrm{H}$ vibrations (from 1300 to $1480 \mathrm{~cm}^{-1}$ ) a slight decrease of the IR bands intensity is observed for the samples prepared with the PVP/Pd ratio 20 but it is quite difficult to see this effect in the other catalysts series due to the small amount of PVP. According to this and the previously reported results, it is possible to understand the diminution of the intensity as an evidence of the PVP dissolution in the methanol suspension. ${ }^{27}$ Another important change is observed over time: the intensity of the band located at $1730 \mathrm{~cm}^{-1}$ decreased after 1 and 6 months. So according to our results the band located at $1730 \mathrm{~cm}^{-1}$ must correspond to a weak interaction (due to its fast disappearance) between an intermediate species of the PVP and the Pd surface.

Figure 3 shows the TEM micrographs of the as-prepared catalysts and their respective histograms obtained from a 10o-particle count. The average NP size of the different fresh samples is $4.8 \pm 1.6$ (with the presence of larger particles as observed in its respective bimodal histogram, in which the majority of the population of NPs are located in the smallest size range), $3.7 \pm 0.7$ and $3.5 \pm 0.7 \mathrm{~nm}$ for the catalysts prepared with a PVP/Pd ratio of 1,10 and 20 , respectively. Coalescence of different NPs was not observed for any of the samples. As it was previously reported, the amount of PVP using during the synthesis of noble metal NPs has an important effect on their size. ${ }^{20,41}$ As it was expected, lower PVP/Pd ratios led to larger NPs, which is particularly evidenced in the case of $\mathrm{PVP} / \mathrm{Pd}=1$, where NPs as large as 9-10 $\mathrm{nm}$ were detected. As it can be seen in the TEM micrographs, the 6-months aged samples did not show any significant change as compared with the fresh catalyst counterparts, neither in NP size nor in morphology. This indicates that the Pd NP suspensions in methanol are stable against the NPs agglomeration and size modification for several months, as reported previously. ${ }^{27}$ As no significant difference in NPs size was ob- 
served after 6 months of the purification step, the catalysts prepared with the 1 month aged NPs were not analysed.

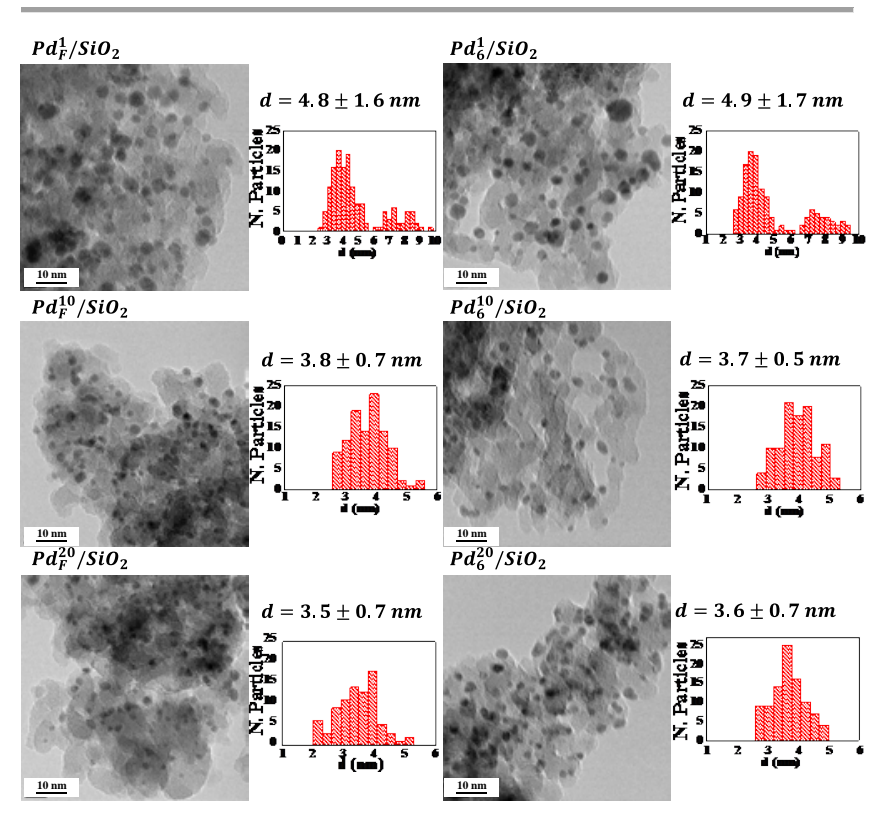

Figure 3. TEM images of the fresh and 6 months aged catalysts together with their corresponding NP size histograms.

Table 2. Results of the catalysts characterisation, in terms of metal loading determined by ICP, average NPs size, $\mathrm{CO}$ adsorption experiments, as well as the electronic state determined by XPS.

\begin{tabular}{|c|c|c|c|c|c|}
\hline \multirow[t]{2}{*}{ Sample } & \multirow{2}{*}{$\begin{array}{l}\text { Metal } \\
\text { loading } \\
(\%)^{[\mathrm{a}]}\end{array}$} & \multirow{2}{*}{$\begin{array}{c}\text { NPs } \\
\text { diame- } \\
\text { ter }(n m)\end{array}$} & \multirow{2}{*}{$\begin{array}{c}\mathrm{CO} \mathrm{ad}^{-} \\
\text {sorption }^{[\mathrm{b}]} \\
(\mathrm{mol} \\
\mathrm{CO} / \mathrm{mol} \\
\mathrm{Pd})\end{array}$} & \multicolumn{2}{|c|}{$\operatorname{Pd}(\%)^{[c]}$} \\
\hline & & & & $\mathrm{Pd}^{\circ}$ & $\mathrm{Pd}^{\delta+}$ \\
\hline $\mathrm{Pd}_{F}^{1} / \mathrm{SiO}_{2}$ & 0.97 & $\begin{array}{c}4.8 \pm \\
1.6\end{array}$ & 0.042 & 72.8 & 27.2 \\
\hline $\mathrm{Pd}_{1}^{1} / \mathrm{SiO}_{2}$ & 1.05 & - & 0.059 & 77.0 & 23.0 \\
\hline $\mathrm{Pd}_{6}^{1} / \mathrm{SiO}_{2}$ & 1.05 & $\begin{array}{c}4.9 \pm \\
1.7\end{array}$ & 0.075 & 80.1 & 19.9 \\
\hline $\mathrm{Pd}_{F}^{10} / \mathrm{SiO}_{2}$ & 1.10 & $\begin{array}{c}3.8 \pm \\
0.7\end{array}$ & 0.016 & $73 \cdot 7$ & 26.3 \\
\hline $\mathrm{Pd}_{1}^{10} / \mathrm{SiO}_{2}$ & 1.00 & - & 0.030 & 81.1 & 18.9 \\
\hline $\mathrm{Pd}_{6}^{10} / \mathrm{SiO}_{2}$ & 0.92 & $\begin{array}{c}3 \cdot 7 \pm \\
0.5\end{array}$ & 0.050 & 83.5 & 16.5 \\
\hline $\mathrm{Pd}_{\mathrm{F}}^{20} / \mathrm{SiO}_{2}$ & 1.01 & $\begin{array}{c}3.5 \pm \\
0.7\end{array}$ & 0.027 & 80.7 & 19.3 \\
\hline $\mathrm{Pd}_{1}^{20} / \mathrm{SiO}_{2}$ & 1.10 & - & 0.040 & 77.8 & 22.2 \\
\hline $\mathrm{Pd}_{6}^{20} / \mathrm{SiO}_{2}$ & 1.09 & $\begin{array}{c}3.6 \pm \\
0.7\end{array}$ & 0.058 & 71.3 & 28.7 \\
\hline
\end{tabular}

${ }^{\text {[a] }}$ Metal loading (wt. \%) calculated by ICP-OES analysis.

${ }^{[b]}$ The Pd normalised results are calculated with ICP-
OES metal loading.

${ }^{\text {[c] }}$ Electronic state of Pd calculated by XPS results.

As it can be seen from the results included in Table 2, the amount of $\mathrm{CO}$ adsorbed is strongly dependent on the PVP/Pd ratio as well as on the NPs aging time. It seems that there is not a very clear trend for the amount of $\mathrm{CO}$ adsorbed by the fresh catalysts with different PVP/Pd ratios, as this adsorption might be affected by several aspects, such as the NP size, electronic state of the Pd atoms in the surface of the NP, as well as by the polymer coverage. In this respect, it would appear that in the case of the sample prepared with the lowest PVP/Pd ratio, the amount of PVP in solution is not enough to surround all the newly formed metal nanoparticles, which in turn results in this bimodal particle size distribution, with the larger particles being thus incompletely coated with PVP molecules. This in turn provides a much more accessible surface for the adsorption of CO molecules, as evidenced from our results in which the samples prepared with a PVP/Pd ratio of 1 present the largest CO adsorption at any given aging time. The evolution of the $\mathrm{CO}$ adsorbed by the samples with the same PVP/Pd ratio and different aging times could provide useful information. In this sense, as the Pd NPs are surrounded by a polymer protective shell, not all Pd surface sites are available and the amount of $\mathrm{CO}$ adsorbed can be considered as an estimation of the accessible Pd sites, irrespective of their being $\mathrm{Pd}^{\circ}$ or $\mathrm{Pd}^{\delta+}$ as it has been previously reported. ${ }^{49,50}$ According to this, the increase in the $\mathrm{CO}$ adsorption with the aging time can be attributed to the PVP dissolution from the NPs surface when the colloid is suspended in methanol (as it has been seen by FTIR) producing an increase of the accessible Pd sites in the NPs surface. Thus, the differences in the adsorbed $\mathrm{CO}$ can be attributed to the polymer coverage/protection of the PVP on the NPs surface and the free accessible Pd.

Regarding the XPS results of the catalysts some properties of the materials can be extracted. On the one hand, XPS allows differentiating between the Pd electronic states in the catalysts. It is reported that the interaction between the metal surface and the carbonyl group of the PVP acts withdrawing electron density from the metal particles surface, which produces an apparent presence of electron deficient states $\left(\right.$ or $\left.\mathrm{Pd}^{\delta+}\right)$. During the synthesis the metal ions dissolved in the ethylene glycol have a strong interaction with the carbonyl groups of the PVP which facilitates the nuclei formation, this interaction remains when the NPs are formed and are responsible for this electron deficient Pd species. ${ }^{7,27,51}$ Pd $3 \mathrm{~d}$ XPS spectra of fresh and 6 months aged catalysts are presented in Figure 4A. In these spectra it is possible to identify two peaks corresponding to the $3 \mathrm{~d}_{5 / 2}$ and $3 \mathrm{~d}_{3 / 2}$ transitions, which can be each deconvoluted into 2 different peaks (resulting in a total of 4 peaks); at 335.5 and $340.4 \mathrm{eV}$, corresponding to the signal of $\mathrm{Pd}^{\circ}$ (green), and at 337.0 and $342.2 \mathrm{eV}$ associated to the presence of $\mathrm{Pd}^{\delta+}$ (black). ${ }^{20}$ In this sense, for all 
the catalysts prepared and analysed in this work, a fraction of $\mathrm{Pd}^{0}$ and $\mathrm{Pd}^{\delta+}$, even for the fresh NPs, is obtained. As it can be seen from the results listed in Table 2, similar $\mathrm{Pd}^{\delta+}$ percentages (close to $27 \%$ ) are obtained for the samples $\mathrm{Pd}_{\mathrm{F}}^{1} / \mathrm{SiO}_{2}$ and $\mathrm{Pd}_{\mathrm{F}}^{10} / \mathrm{SiO}_{2}$ and the small differences observed (probably due to the experimental error) might indicate that a similar interaction between the carbonyl group of the PVP and the Pd NPs surface takes place in these samples. However, an unexpected value is observed for the sample prepared with higher PVP/Pd molar ratio $\left(\mathrm{Pd}_{\mathrm{F}}^{20} / \mathrm{SiO}_{2}\right)$. It was expected that the higher PVP content in this sample favored the Pd-carbonyl interactions, leading to the highest $\mathrm{Pd}^{\delta+} \%$, as a consequence of the electron density transfer from Pd NPs surface to the $\mathrm{CO}$ group. However, the $\mathrm{Pd}^{\delta+}$ content in $\mathrm{Pd}_{F}^{20} / \mathrm{SiO}_{2}$ is the lowest among the fresh samples investigated ( $19 \%)$. These results might indicate that additional effects should be considered when analysing the electronic features of the studied Pd NPs.

The observation of the Pd $3 d$ XPS spectra of samples $\mathrm{Pd}^{20} / \mathrm{SiO}_{2}$ (Figure $4 \mathrm{~A}$ ) revealed a significant displacement of the $3 \mathrm{~d}_{5 / 2}$ transition peak to lower binding energies (BE) for this fresh sample as compared to $\mathrm{Pd}_{F}^{1} / \mathrm{SiO}_{2}$ and $\mathrm{Pd}_{F}^{10} /$ $\mathrm{SiO}_{2}$, indicating that the electronic features of the samples prepared with the highest PVP content are different from those in the $\mathrm{Pd}^{1} / \mathrm{SiO}_{2}$ and $\mathrm{Pd}^{10} / \mathrm{SiO}_{2}$ series of catalysts. In order to get a better insight into the PVP-metal interaction, the $\mathrm{N}$ is spectra are shown in Figure ${ }_{4} \mathrm{~B}$. As it can be seen, samples $\mathrm{Pd}^{1} / \mathrm{SiO}_{2}$ and $\mathrm{Pd}^{10} / \mathrm{SiO}_{2}$ showed only one peak located at $399.9 \mathrm{eV}$ ascribed to the pure pyrrolidone groups, ${ }^{8}$ indicating that the $\mathrm{N}$ groups do not interact with the Pd surface or their interaction does not modify the $\mathrm{N}$ electronic properties. However, for the catalysts prepared with the highest PVP/Pd ratio (20), a new peak is observed at higher binding energies $(400.9 \mathrm{eV})$ for both fresh and aged NPs, which might be correlated to the presence of electron deficient $\mathrm{N}$ groups. ${ }^{8,21}$ This fact revealed that when a high PVP content is used in the Pd NPs synthesis $(\mathrm{PVP} / \mathrm{Pd}=\mathbf{2 0})$, the polymer molecules do not only interact with the Pd surface through the $\mathrm{CO}$ groups by withdrawing electron density from the metal surface, but there is also a charge transfer from the $\mathrm{N}$ groups of the PVP molecules to the metal surface (by bridged interaction but without the PVP-ring breakage $(401.6 \mathrm{eV})){ }^{8}$, which results in the electron density Pd enrichment (in $\mathrm{Pd}^{\circ}$ content and XPS Pd peak displacement to lower $\mathrm{BE}$ ) observed in the fresh $\mathrm{Pd}^{20} / \mathrm{SiO}_{2}$ as compared to and $\mathrm{Pd}^{1} / \mathrm{SiO}_{2}$ and $\mathrm{Pd}^{10} / \mathrm{SiO}_{2}{ }^{8,9}$

Another important aspect to be considered is the evolution of the relative proportion of $\mathrm{Pd}^{\circ}$ with the aging time for the different PVP/Pd ratios under study. In this sense, when the aging time increases, an increase of the $\mathrm{Pd}^{\circ}$ percentage in observed for the catalysts prepared with the $\mathrm{PVP} / \mathrm{Pd}$ ratio of 1 and 1o. This effect is in good agreement with the previously reported study ${ }^{27}$ and it is a clear result of the PVP dissolution in the methanol solvent when the NPs colloids are stored before their loading onto the support. When the PVP is progressively removed from the NPs surface, the relative proportion of $\mathrm{Pd}^{\circ}$ increases as a consequence of the undermined electronic transfer from the Pd surface to the carbonyl groups of the polymer molecules.

However, for the highest PVP/Pd ratio studied (20), the opposite effect is observed. In this set of catalysts the $\mathrm{Pd}^{\circ}$ (or electron rich metal specie) amount decreases progressively from $80.7 \%$ in the fresh samples to $71.3 \%$ in the 6 months aged sample. This decrease in the electron rich Pd content agrees well with the PVP dissolution in the colloid with the aging time (as the $\mathrm{N}$ groups responsible of the electronic transfer to the Pd surface are progressively detached from the metal surface) then removing essentially the interaction with the carbonyl groups.

On the other hand, the N/Pd ratio of the samples can be obtained analysing the quantitative XPS results of the $\mathrm{N}$ and the Pd. The different N/Pd ratios calculated from XPS analysis shows the amount of the PVP used in the synthesis and its progressive dissolution of the PVP in the methanol suspension. As it was expected, for the fresh samples, the N/Pd ratio increases with the PVP/Pd ratio used in the colloids synthesis $(2.3,4.2$ and 10.8 respectively from the $\mathrm{Pd}_{\mathrm{F}}^{1} / \mathrm{SiO}_{2}$ to the $\mathrm{Pd}_{F}^{20} / \mathrm{SiO}_{2}$ catalysts). When the fresh catalysts are compared with their 6 months aged counterparts, a decrease in the N/Pd ratio is observed for the three sets of catalysts (1.4, 3.2 and 5.8, for PVP/Pd of 1,10 and 20, respectively). This effect is also an evidence of the previously reported results of the partial dissolution of the PVP in methanol along the time after the purification of the NPs. ${ }^{27}$

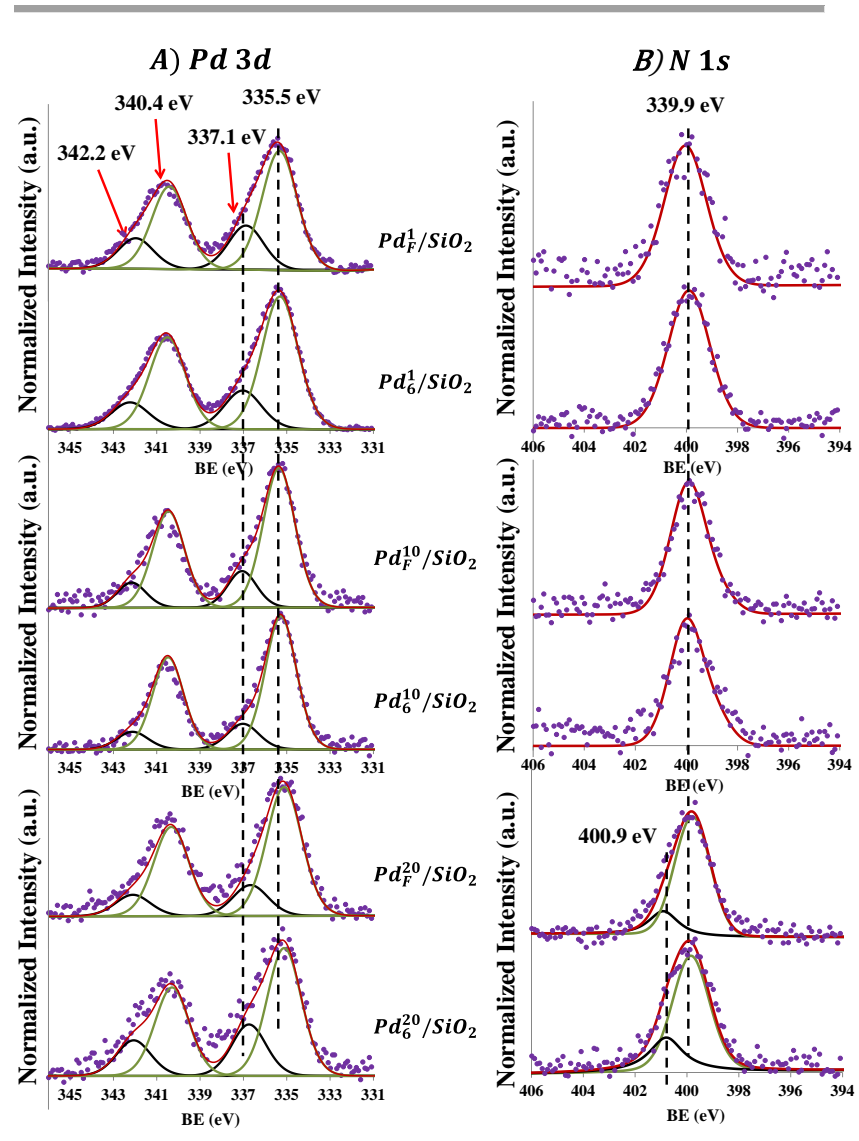


Figure 4. A) Pd (3d) and B) N (1s) spectra of the fresh and 6 months aged catalysts.

Once the catalytic results in FA decomposition and the most relevant characterisation were presented, a critical discussion of the results is included in this section. As it was claimed previously, a very strong dependence between the catalytic FA decomposition reaction and the evolution of the PVP and Pd NPs over time in the colloid suspension in methanol is observed. In this sense, there are some effects, analysed in this work, which may be very influential in the FA decomposition due to the modification of the Pd surface features. These effects can be distinguished and summarised as follows:

i) Some studies emphasized the problem of PVP coverage and Pd accessibility of the NPs surface when PVP is used as capping agent for the protection/stabilisation of the noble metal NPs during their synthesis. ${ }^{11,13,24}$ The coating of the polymer around the NP prevents their agglomeration and is also responsible for the reduction of its available surface due to the partial blocking of possible active sites in the reaction. However, the beneficial effect of the PVP presence on the NPs surface must be taken into account. ${ }^{9,24,25}$ Some authors reported that the PVP anchored on the surface of the NPs can increase the specific catalytic activity of the free $\mathrm{Pd}$ active sites. In this sense, it was addressed that, in some cases, the specific catalytic ability of the Pd sites is inversely proportional to the $\mathrm{Pd}$ accessibility (i.e. lower $\mathrm{Pd}$ accessibility resulted in few Pd active sites with strengthened catalytic ability). ${ }^{24}$ According to previous studies, ${ }^{37,39}$ the FA dehydrogenation reaction mechanism goes through a palladium-formate $\left(\mathrm{Pd}-[\mathrm{HCOO}]^{-}\right)$intermediate, and therefore the catalytic performance is strongly dependent on the Pd surface features. The Pd surface deactivation due to the occupation of active sites by reaction intermediates compounds (such as $\mathrm{H}^{+}, \mathrm{CO}_{2}, \mathrm{H}_{2} \mathrm{O}$ and/or $\mathrm{HCOO}^{-}$) has been also studied by Hu et al.. ${ }^{52}$ In this sense, the increase in Pd accessibility due to PVP dissolution might favor the adsorption of intermediates on the $\mathrm{Pd}$ surface, leading to space exclusion and repulsive adsorbate-adsorbate interaction that would finally decline the catalytic ability in the $\mathrm{H}_{2}$ production.

ii) The NP size must also be taken into account in order to compare the catalytic activity in FA decomposition tests. Recently, the relationship between the Pd NP size (supported on an activated carbon material) and the $\mathrm{H}_{2}$ output by FA decomposition has been described as a volcano plot between 2.7 and $5.5 \mathrm{~nm}$ of NPs average size, observing the highest activity in the FA decomposition for the Pd NPs with an average size of $3.9 \mathrm{~nm}^{.11}$

iii) The electronic state of the Pd is a very important parameter in the FA decomposition. In all cases, the prereduction of the catalysts when the $\mathrm{Pd}$ is added to the support is mandatory to obtain Pd in its metallic form, which is the active state of Pd in the catalytic decomposition of the FA..$^{38,53} \mathrm{~A}$ different approach studied in our research group was the alloying of Pd with metals with lower ionization potential, such as $\mathrm{Ag}$ or $\mathrm{Cu}$, so as the resulting Pd species was electronically enriched and an enhancement in the activity compared with the nonalloyed metal was observed..$^{37,40}$

In our work, all these effects are interconnected and in turn related to the PVP presence in the colloidal synthesis. Furthermore, with the already mentioned PVP dissolution in the methanol colloid along the time all these effects can be assessed in the catalysts prepared with the same NPs.

Regarding the Pd accessibility effect, the relationship between the $\mathrm{CO}$ adsorbed on the Pd surface and the initial TOF values achieved with the three sets of catalysts is displayed in Figure 5.

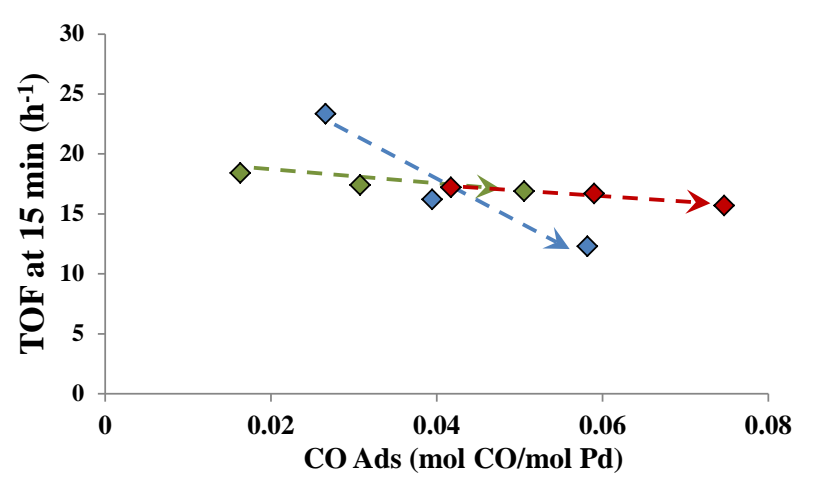

Figure 5. TOF values vs the adsorbed CO. Red dots correspond to $\boldsymbol{P} \boldsymbol{d}^{\mathbf{1}}$, green to the $\boldsymbol{P} \boldsymbol{d}^{\mathbf{1 0}}$, and blue for the catalysts prepared with the nanoparticles $\boldsymbol{P} \boldsymbol{d}^{\mathbf{2 0}}$. The colored arrows indicate the NPs aging time increase in the methanol solution.

As it can be seen, a slight trend is obtained for the samples prepared with the NPs $P d^{1}$ and $P d^{10}$. As the Pd accessibility (in terms of $\mathrm{CO}$ adsorbed) on the NPs surface increases, the initial catalytic activity (expressed as initial TOF) of the catalysts decreases. For the catalysts with the highest PVP content, this effect is more pronounced and when the adsorbed CO increased from 0.027 to $0.058 \mathrm{~mol}$ $\mathrm{CO} / \mathrm{mol} \mathrm{Pd}$, the TOF value decreased considerably (from 23.4 to $12.3 \mathrm{~h}^{-1}$ ). This effect is in good agreement with the beneficial effect of the use of PVP as capping agent, even more so compared with the uncapped-Pd impregnated catalysts as reported elsewhere for other reactions., ${ }^{90,24,25}$

According to NPs size, TEM analysis indicated that the $\mathrm{PVP} / \mathrm{Pd}$ ratio used in the NP synthesis has an important effect on the NPs size $(4.8,3.7$ and $3.5 \mathrm{~nm}$ for PVP/Pd ratios of 1,10 and 20 , respectively). The better catalytic performance of samples with average NP size of 3.5 and 3.7 as compared with larger ones is in good agreement with our recent study. ${ }^{41}$ However, as the NPs preserved their size and morphology over time, any effect which the NPs size may have in the catalytic activity decay towards $\mathrm{H}_{2}$ production when aged NPs were used can be ruled 
out. In this case the effect of other features might become more relevant.

Upon consideration of the catalytic test results as well as the characterisation of the catalysts, it is clear that the surface electronic features of Pd NPs might be a key point to be considered. As it was previously discussed, an increase of $\mathrm{Pd}^{\mathrm{o}}$ content is observed over time for samples prepared with $\mathrm{PVP} / \mathrm{Pd}=1$ and 10 , while a reduction of the $\mathrm{Pd}^{\circ}$ proportion can be clearly appreciated for samples with $\mathrm{PVP} / \mathrm{Pd}=\mathbf{2 0}$ (results presented in Table $\mathbf{2}$ ). According to the catalytic activity decay in the FA decomposition tests (See Figure 1 and Table 1 ) and the catalysts characterisation results and previous discussion, a possible interpretation of the different catalytic activity decays can be proposed.

In case of the catalysts prepared with the NPs with a $\mathrm{PVP} / \mathrm{Pd}$ ratio of 1 and 10, a decrease of the activity around $22 \%$ (in terms of $\mathrm{H}_{2}$ output) and $8 \%$ (in TOF values) after 6 months of the NPs purification step are observed. For these samples, the dissolution of PVP in the colloids with the NPs aging led to an increase of $\mathrm{Pd}^{\circ}$ percentage (as a consequence of the undermined electronic charge transfer from the NPs surface to the $\mathrm{CO}$ groups of the PVP molecules) together with an increase of the Pd accessibility. Then, the slight catalytic activity decay observed in these two sets of samples might be due to both compensated effects. It was expected that the increase of surface electron rich $\mathrm{Pd}$ species (expressed as $\mathrm{Pd}^{\mathrm{o}}$ content) with the time would have a positive effect on the catalytic ability towards the $\mathrm{H}_{2}$ production, however, the increase of the Pd accessibility observed for the aged samples might favor the adsorption of reaction intermediates, which could lead to space exclusion and repulsive adsorbate-adsorbate interaction that would ultimately decline the catalytic ability in the $\mathrm{H}_{2}$ production. It should be noticed at this point that the negative effect of the surface $\mathrm{Pd}$ accessibility increase can be evidenced by the comparison of the activity displayed by samples $\mathrm{Pd}_{\mathrm{F}}^{1} / \mathrm{SiO}_{2}$ and $\mathrm{Pd}_{F}^{10} / \mathrm{SiO}_{2}$ (with similar $\mathrm{Pd}^{\mathrm{o}}$ content). Along these lines, the better accessibility shown by sample $\mathrm{Pd}_{F}^{1} / \mathrm{SiO}_{2}$ as compared with sample $\mathrm{Pd}_{\mathrm{F}}^{10} / \mathrm{SiO}_{2}$ (0.042 and $0.016 \mathrm{~mol}$ $\mathrm{CO} / \mathrm{mol} \mathrm{Pd}$, respectively) resulted in a poorer catalytic activity in terms of $\mathrm{H}_{2}$ production (151.7 and $186.5 \mu \mathrm{mol}$ of $\mathrm{H}_{2}$ after 180 min of reaction for $\mathrm{Pd}_{F}^{1} / \mathrm{SiO}_{2}$ and $\mathrm{Pd}_{\mathrm{F}}^{10} / \mathrm{SiO}_{2}$, respectively).

Then, for both series of samples, the studied Pd surface features ( $\mathrm{Pd}^{\circ}$ content and $\mathrm{Pd}$ surface accessibility) are partially compensated and result in a final catalytic activity decay of around $22 \%$ in both sets.

In the case of the catalysts prepared with the $P d^{20} \mathrm{NPs}$, a marked reduction of the catalytic ability was observed (61.5\% of its initial activity in terms of $\mathrm{H}_{2}$ generation and $50 \%$ of TOF reduction after 6 months). As in the other samples prepared with lower PVP contents, the PVP dissolution along the time produces an increase of the $\mathrm{Pd}$ accessibility, with the subsequent aforementioned possible steric hindrance drawbacks. However, in these samples the negative effect of the $\mathrm{Pd}^{\mathrm{o}}$ content decrease with the aging time (See Table 2 ) might be an additional negative factor in declining the ability of the PVP-Pd catalytic system towards the $\mathrm{H}_{2}$ production from FA dehydrogenation, resulting in the marked activity decay of around $60 \%$ after 6 months.

\section{CONCLUSIONS}

Catalysts based on $\mathrm{SiO}_{2}$ supported PVP-capped Pd NPs prepared by reduction by solvent methodology were tested in the FA decomposition reaction in liquid-phase. This study is focused on the PVP protection effect (with 3 different PVP/Pd synthesis ratios) and their evolution over time after the purification step, as well as on their effect in the final catalytic performance. With spectroscopy techniques as XPS or FTIR and CO adsorption experiments, the PVP dissolution once the colloids were redispersed in methanol was evidenced. The progressive dissolution of the PVP in the methanol suspension after 6 months did not have any effect in the Pd NPs size and morphology, but it was proven to have a very strong influence on the Pd NP surface features, in terms of both surface electronic properties and Pd sites accessibility. The assessment of both characteristics allowed us to elucidate the pivotal role of PVP molecules in affording active and stable $\mathrm{Pd}$-based catalysts towards the $\mathrm{H}_{2}$ production from FA dehydrogenation. The results herein reported will be a useful tool for the future design of highly efficient polymer capped-metal nanoparticles with optimised performances in the $\mathrm{H}_{2}$ production from $\mathrm{H}_{2}$ storage/release materials.

\section{AUTHOR INFORMATION}

\section{Corresponding Author}

Prof. D. Cazorla-Amorós (e-mail: cazorla@ua.es).

Prof. H. Yamashita (e-mail: yamashita@mat.eng.osakau.ac.jp).

\section{ACKNOWLEDGMENT}

We thank the Spanish Ministry of Economy and Competitiveness (MINECO), Generalitat Valenciana and FEDER (Projects CTQ2015-66o8o-R MINECO/FEDER and PROMETEOII/2014/o10) for financial support. J.G.A. thanks the MINECO for his fellowship (BES-2013-063678) and specially thanks the mobility grant of MINECO, EEBB-I-15-10219 at Osaka University.

\section{REFERENCES}

(1) Niu, Z.; Li, Y. Removal and Utilization of Capping Agents in Nanocatalysis. Chem. Mater. 2014, 26, 72-83. Koczkur, K. M.; Mourdikoudis, S.; Polavarapu, 
L.; Skrabalak, S. E. Polyvinylpyrrolidone (PVP) in Nanoparticle Synthesis. Dalt. Trans. 2015, 17883-17905.

(3) Narayanan, R.; El-Sayed, M. A. ShapeDependent Catalytic Activity of Platinum Nanoparticles in Colloidal Solution. Nano Lett. 2004, 4, 1343-1348.

(4) Rogers, S. M.; Catlow, C. R. A.; Chan-Thaw, C. E.; Gianolio, D.; Gibson, E. K.; Gould, A. L.; Jian, N.; Logsdail, A. J.; Palmer, R. E.; Prati, L.; et al. Tailoring Gold Nanoparticle Characteristics and the Impact on Aqueous-Phase Oxidation of Glycerol. ACS Catal. 2015, 5, 4377-4384.

(5) Shao, M.; Yu, T.; Odell, J. H.; Jin, M.; Xia, Y. Structural Dependence of Oxygen Reduction Reaction on Palladium Nanocrystals. Chem. Commun. 2011, 47, 6566-6568.

(6) Wang, Y.; Zheng, Y.; Huang, C. Z.; Xia, Y. Synthesis of Ag Nanocubes 18-32 Nm in Edge Length: The Effects of Polyol on Reduction Kinetics, Size Control, and Reproducibility. J. Am. Chem. Soc. 2013, 135, 1941-1951.

(7) Borodko, Y.; Habas, S. E.; Koebel, M.; Yang, P.; Frei, H.; Somorjai, G. A. Probing the Interaction of Poly(vinylpyrrolidone) with Platinum Nanocrystals by UV - Raman and FTIR. J. Phys. Chem. B 2006, 110, 23052-23059.

(8) Collins, G.; Schmidt, M.; McGlacken, G. P.; O’Dwyer, C.; Holmes, J. D. Stability, Oxidation, and Shape Evolution of PVP-Capped Pd Nanocrystals. J. Phys. Chem. C 2014, 118, 65226530.

(9) Tsunoyama, H.; Ichikuni, N.; Sakurai, H.; Tsukuda, T. Effect of Electronic Structures of $\mathrm{Au}$ Clusters Stabilized by poly(N-Vinyl-2Pyrrolidone) on Aerobic Oxidation Catalysis. J. Am. Chem. Soc. 2009, 131, 7086-7093.

(10) Villa, A.; Wang, D.; Su, D. S.; Prati, L. Gold Sols as Catalysts for Glycerol Oxidation: The Role of Stabilizer. ChemCatChem 2009, 1, 510-514.

(11) Quintanilla, A.; Butselaar-Orthlieb, V. C. L.; Kwakernaak, C.; Sloof, W. G.; Kreutzer, M. T.; Kapteijn, F. Weakly Bound Capping Agents on Gold Nanoparticles in Catalysis: Surface Poison? J. Catal. 2010, 271, 104-114.

(12) Morsbach, E.; Nesselberger, M.; Warneke, J.; Harz, P.; Arenz, M.; Bäumer, M.; Kunz, S. 1Naphthylamine Functionalized $\mathrm{Pt}$ Nanoparticles: Electrochemical Activity and Redox Chemistry Occurring on One Surface. New J. Chem. 2015, 39, 2557-2564.

(13) Zhao, Y.; Jia, L.; Medrano, J. A.; Ross, J. R. H.; Le, L. Supported Pd Catalysts Prepared via Colloidal Method: The Effect of Acids. ACS
Catal. 2013, 3, 2341-2352.

(14) Hirai, H.; Yakura, N. Protecting Polymers in Suspension of Metal Nanoparticles. Polym. Adv. Technol. 2001, 12, 724-733.

(15) He, B.; Ha, Y.; Liu, H.; Wang, K.; Liew, K. Y. Size Control Synthesis of Polymer-Stabilized Water-Soluble Platinum Oxide Nanoparticles. J. Colloid Interface Sci. 2007, 308, 105-111.

(16) Xu, Y.; Chen, L.; Wang, X.; Yao, W.; Zhang, Q. Recent Advances in Noble Metal Based Composite Nanocatalysts: Colloidal Synthesis, Properties, and Catalytic Applications. Nanoscale 2015, 7, 10559-10583.

(17) Borodko, Y.; Humphrey, S. M.; Tilley, T. D.; Frei, H.; Somorjai, G. A. Charge-Transfer Interaction of Poly(vinylpyrrolidone) with Platinum and Rhodium Nanoparticles. J. Phys. Chem. C 2007, 111, 6288-6295.

(18) Wu, C.; Mosher, B. P.; Lyons, K.; Zeng, T. Reducing Ability and Mechanism for Polyvinylpyrrolidone (PVP) in Silver Nanoparticles Synthesis. J. Nanosci. Nanotechnol. 2010, 10, 2342-2347.

(19) Ke, F.; Wang, L.; Zhu, J. An Efficient Room Temperature Core-shell AgPd@MOF Catalyst for Hydrogen Production from Formic Acid. Nanoscale 2015, 7, 8321-8325.

(20) Miguel-García, I.; Berenguer-Murcia, Á.; Cazorla-Amorós, D. Preferential Oxidation of CO Catalyzed by Supported Polymer-Protected Palladium-Based Nanoparticles. Appl. Catal. B Environ. 2010, 98, 161-170.

(21) Xian, J.; Hua, Q.; Jiang, Z.; Ma, Y.; Huang, W. Size-Dependent Interaction of the Poly $(\mathrm{N}$ Vinyl-2-Pyrrolidone) Capping Ligand with $\mathrm{Pd}$ Nanocrystals. Langmuir 2012, 28, 6736-6741.

(22) Evangelisti, C.; Panziera, N.; D’Alessio, A.; Bertinetti, L.; Botavina, M.; Vitulli, G. New Monodispersed Palladium Nanoparticles Stabilized by Poly-(N-Vinyl-2-Pyrrolidone): Preparation, Structural Study and Catalytic Properties. J. Catal. 2010, 272, 246-252.

(23) Qiu, L.; Liu, F.; Zhao, L.; Yang, W.; Yao, J. Evidence of a Unique Electron Donor Acceptor Property for Platinum Nanoparticles as Studied by XPS. Langmuir 2006, 22, 44804482.

(24) Zhao, Y.; Baeza, J. A.; Koteswara Rao, N.; Calvo, L.; Gilarranz, M. A.; Li, Y. D.; Lefferts, L. Unsupported PVA- and PVP-Stabilized Pd Nanoparticles as Catalyst for Nitrite Hydrogenation in Aqueous Phase. J. Catal. 2014, 318, 162-169.

(25) Sonström, P.; Arndt, D.; Wang, X.; Zielasek, V.; 
Bäumer, M. Ligand Capping of Colloidally Synthesized Nanoparticles-AWay to Tune Metal-Support Interactions in Heterogeneous Gas-Phase Catalysis. Angew. Chem. Int. Ed. Engl. 2011, 50, 3888-3891.

(26) Li, Y.; El-Sayed, M. A. The Effect of Stabilizers on the Catalytic Activity and Stability of Pd Colloidal Nanoparticles in the Suzuki Reactions in Aqueous Solution. J. Phys. Chem. B 2001, 105, 8938-8943.

(27) Miguel-García, I.; Berenguer-Murcia, Á.; García, T.; Cazorla-Amorós, D. Effect of the Aging Time of PVP Coated Palladium Nanoparticles Colloidal Suspensions on Their Catalytic Activity in the Preferential Oxidation of CO. Catal. Today 2012, 187, 2-9.

(28) Tedsree, K.; Li, T.; Jones, S.; Chan, C. W. A.; Yu, K. M. K.; Bagot, P. A. J.; Marquis, E. a; Smith, G. D. W.; Tsang, S. C. E. Hydrogen Production from Formic Acid Decomposition at Room Temperature Using a Ag-Pd Core-Shell Nanocatalyst. Nat. Nanotechnol. 2011, 6, 302307.

(29) Liao, F.; Lo, T. W. B.; Tsang, S. C. E. Recent Developments in Palladium-Based Bimetallic Catalysts. ChemCatChem 2015, 7, 1998-2014.

(30) Dalebrook, A. F.; Gan, W.; Grasemann, M.; Moret, S.; Laurenczy, G. Hydrogen Storage: Beyond Conventional Methods. Chem. Commun. 2013, 49, 8735-8751.

(31) Grasemann, M.; Laurenczy, G. Formic Acid as a Hydrogen Source - Recent Developments and Future Trends. Energy Environ. Sci. 2012, 5, 8171-8181.

(32) Roduner, E. Understanding Catalysis. Chem. Soc. Rev. 2014, 43, 8226-8239.

(33) Zacharska, M.; Podyacheva, O. Y.; Kibis, L. S.; Boronin, A. I.; Senkovskiy, B. V.; Gerasimov, E. Y.; Taran, O. P.; Ayusheev, A. B.; Parmon, V. N.; Leahy, J. J.; et al. Ruthenium Clusters on Carbon Nanofibers for Formic Acid Decomposition: Effect of Doping the Support with Nitrogen. ChemCatChem 2015, 7, 29102917.

(34) Enthaler, S.; von Langermann, J.; Schmidt, T. Carbon Dioxide and Formic Acid-the Couple for Environmental-Friendly Hydrogen Storage? Energy Environ. Sci. 2010, 3, 1207-1217.

（35） Bulushev, D. A.; Beloshapkin, S.; Ross, J. R. H. Hydrogen from Formic Acid Decomposition over Pd and Au Catalysts. Catal. Today 2010, 154, 7-12.

(36) Zhang, S.; Metin, Ö.; Su, D.; Sun, S. Monodisperse AgPd Alloy Nanoparticles and
Their Superior Catalysis for the Dehydrogenation of Formic Acid. Angew. Chemie - Int. Ed. 2013, 52, 3681-3684.

(37) Mori, K.; Dojo, M.; Yamashita, H. Pd and Pd-Ag Nanoparticles within a Macroreticular Basic Resin: An Efficient Catalyst for Hydrogen Production from Formic Acid Decomposition. ACS Catal. 2013, 3, 1114-1119.

(38) Martis, M.; Mori, K.; Fujiwara, K.; Ahn, W. S.; Yamashita, H. Amine-Functionalized MIL-125 with Imbedded Palladium Nanoparticles as an Efficient Catalyst for Dehydrogenation of Formic Acid at Ambient Temperature. J. Phys. Chem. C 2013, 117, 22805-22810.

(39) Navlani-García, M.; Martis, M.; LozanoCastelló, D.; Cazorla-Amorós, D.; Mori, K.; Yamashita, $\mathrm{H}$. Investigation of $\mathrm{Pd}$ Nanoparticles Supported on Zeolites for Hydrogen Production from Formic Acid Dehydrogenation. Catal. Sci. Technol. 2015, 5, 364-371.

(40) Mori, K.; Tanaka, H.; Dojo, M.; Yoshizawa, K.; Yamashita, H. Synergic Catalysis of PdCu Alloy Nanoparticles within a Macroreticular Basic Resin for Hydrogen Production from Formic Acid. Chem. - A Eur. J. 2015, 21, 12085-12092.

(41) Navlani-García, M.; Mori, K.; Nozaki, A.; Kuwahara, Y.; Yamashita, H. Investigation of Size Sensitivity in the Hydrogen Production from Formic Acid over Carbon-Supported Pd Nanoparticles. ChemistrySelect 2016, 1, 18791886.

(42) Miguel-García, I.; Navlani-García, M.; GarcíaAguilar, J.; Berenguer-Murcia, Á.; LozanoCastelló, D.; Cazorla-Amorós, D. Capillary Microreactors Based on Hierarchical $\mathrm{SiO}_{2}$ Monoliths Incorporating Noble Metal Nanoparticles for the Preferential Oxidation of CO. Chem. Eng. J. 2015, 275, 71-78.

(43) García-Aguilar, J.; Miguel-García, I.; BerenguerMurcia, Á.; Cazorla-Amorós, D. Synthesis of Robust Hierarchical Silica Monoliths by Surface-Mediated Solution/Precipitation Reactions over Different Scales: Designing Capillary Microreactors for Environmental Applications. ACS Appl. Mater. Interfaces 2014, 6, 22506-22518.

(44) García-Aguilar, J.; Miguel-García, I.; BerenguerMurcia, Á.; Cazorla-Amorós, D. Single Wall Carbon Nanotubes Loaded with Pd and NiPd Nanoparticles for $\mathrm{H}_{2}$ Sensing at Room Temperature. Carbon 2014, 66, 599-611.

(45) Navlani-García, M.; Miguel-García, I.; Berenguer-Murcia, Á.; Lozano-Castelló, D.; 
Cazorla-Amorós, D.; Yamashita, H. Pd/Zeolite-

Based Catalysts for the Preferential CO Oxidation Reaction: Ion-Exchange, $\mathrm{Si} / \mathrm{Al}$ and Structure Effect. Catal. Sci. Technol. 2016.

(46) Domínguez-Domínguez, S.; Berenguer-Murcia, Á.; Cazorla-Amorós, D.; Linares-Solano, Á. Semihydrogenation of Phenylacetylene Catalyzed by Metallic Nanoparticles Containing Noble Metals. J. Catal. 2006, 243, 74-81.

(47) García-Aguilar, J.; Miguel-García, I.; BerenguerMurcia, A.; Cazorla-Amorós, D. Synthesis of Robust Hierarchical Silica Monoliths by Surface- Mediated Solution/Precipitation Reactions over Different Scales: Designing Capillary Microreactors for Environmental Applications. ACS Appl. Mater. Interfaces 2014, 6, 22506-22518.

(48) McCool, B.; Murphy, L.; Tripp, C. P. A Simple FTIR Technique for Estimating the Surface Area of Silica Powders and Films. J. Colloid Interface Sci. 2006, 295, 294-298.

(49) Fernández-García, M.; Martínez-Arias, A.; Salamanca, L. N.; Coronado, J. M.; Anderson, J. A.; Conesa, J. C.; Soria, J. Influence of Ceria on Pd Activity for the $\mathrm{CO}+\mathrm{O}_{2}$ Reaction. J. Catal.
1999, 187, 474-485.

(50) Zhu, H.; Qin, Z.; Shan, W.; Shen, W.; Wang, J. $\mathrm{Pd} / \mathrm{CeO}_{2}-\mathrm{TiO}_{2}$ Catalyst for $\mathrm{CO}$ Oxidation at Low Temperature: A TPR Study with $\mathrm{H}_{2}$ and $\mathrm{CO}$ as Reducing Agents. J. Catal. 2004, 225, 267-277.

(51) Grass, M. E.; Joo, S. H.; Zhang, Y.; Somorjai, G. A. Colloidally Synthesized Monodisperse $\mathrm{Rh}$ Nanoparticles Supported on SBA-15 for Sizeand Pretreatment-Dependent Studies of CO Oxidation. J. Phys. Chem. C 2009, 113, 86168623.

(52) Hu, C.; Pulleri, J. K.; Ting, S. W.; Chan, K. Y. Activity of $\mathrm{Pd} / \mathrm{C}$ for Hydrogen Generation in Aqueous Formic Acid Solution. Int. J. Hydrogen Energy 2014, 39, 381-390.

（53） Song, F.-Z.; Zhu, Q.-L.; Tsumori, N.; Xu, Q. Diamine-Alkalized Reduced Graphene Oxide: Immobilization of Sub-2 Nm Palladium Nanoparticles and Optimization of Catalytic Activity for Dehydrogenation of Formic Acid. ACS Catal. 2015, 5, 5141-5144. 


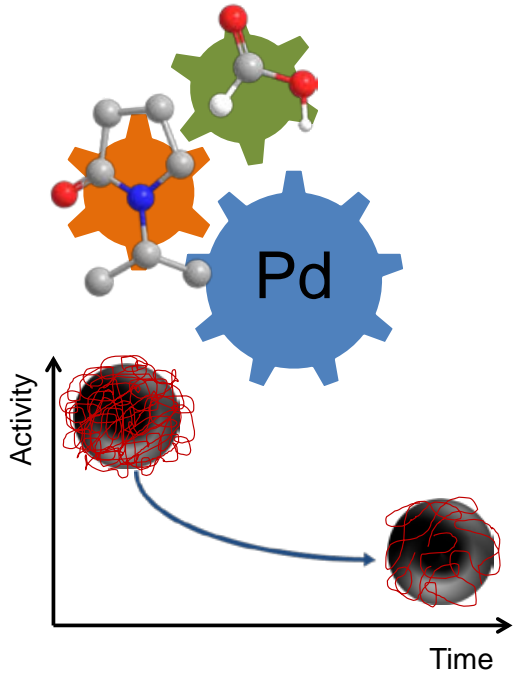

